\title{
Phase transfer and point-spread function of the human eye determined by a new asymmetric double-pass method
}

\author{
Rafael Navarro and M. Angeles Losada \\ Instituto de Optica, Daza de Valdés, Consejo Superior de Investigaciones Científicas, \\ Serrano 121, 28006 Madrid, Spain
}

Received December 1, 1994; accepted June 14, 1995; revised manuscript received June 26, 1995

\begin{abstract}
A recent study has shown that the double-pass method provides a good estimate of the ocular modulation transfer function (MTF) but that it does not yield the phase transfer function (PTF) [J. Opt. Soc. Am. A 12, 195 (1995)]. Therefore, one cannot recover the true retinal point-spread function (PSF). We present a modification of the double-pass method to overcome this problem. The key is to break the symmetry between the two passes. By using an unexpanded Gaussian input beam, we produce a diffraction-limited PSF for the first pass. Then, by using a large exit pupil, we get an aberrated PSF for the second pass. The double-pass aerial image is the cross correlation of both PSF's, so that the Fourier transform of such an aerial image directly provides the true retinal PTF, up to the cutoff frequency of the effective (small), diffraction-limited entrance pupil. The resulting double-pass aerial image is a blurred version of the true retinal PSF. Thus it shows the effect not only of even symmetric aberrations but also of odd and irregular aberrations such as coma. We have explored two different ways to retrieve the true retinal PSF: (a) deblurring of the aerial image and (b) PSF reconstruction combining PTF data with conventional double-pass MTF. We present promising initial results with both artificial and real eyes. (c) 1995 Optical Society of America
\end{abstract}

\section{INTRODUCTION}

The double-pass method ${ }^{1}$ has proved to be one of the most useful ways to measure the retinal image quality of the human eye. It provides, in a direct way, good estimates of the ocular modulation transfer function (MTF) ${ }^{2-4}$ Recent comparative studies have provided a good match between double-pass MTF and MTF's obtained with other methods, including the psychophysical interferometric technique ${ }^{5}$ and computations from wave aberration data ${ }^{6}$ (measured by a Hartmann-Shack wave-front sensor ${ }^{7}$ ). However, a recent study ${ }^{8}$ has shown that the double-pass aerial image of a point test is not its autoconvolution but its autocorrelation. Consequently, the double-pass method cannot provide information about the phase transfer function (PTF). Therefore we cannot retrieve the true single-pass retinal point-spread function (PSF) from the double-pass aerial image. Another important consequence of the loss of phase information is that those aberrations with odd symmetry become even after the second pass (coma, irregular, etc.). The double pass is one of the three main methods used to assess the optical quality of the eye, which correspond to the three basic ways to describe the quality of optical systems. Imaging gratings is the most direct or natural way to obtain the optical transfer function (OTF). For the eye, interferometric methods ${ }^{5}$ can provide accurate estimates of the ocular MTF but not of the PTF, which is also necessary to obtain the PSF. Different wave-front sensors have successfully provided the eye's wave aberration. ${ }^{7,9}$ From these data (and knowing the pupil transmission), one can estimate both the OTF, including the $\mathrm{PTF},{ }^{10}$ and the PSF. However, since the most direct and natural way to measure the PSF of an optical system is imaging a point object, it is important to overcome the limitation (phase loss) of the double-pass method.
In this paper we show how to keep information of the PTF by breaking the symmetry of the double pass, using different entrance and exit pupil sizes. The idea is to make the entrance pupil small (diffraction limited) and use a large exit pupil. The cross correlation between an image (aberrated or not) with a diffraction-limited PSF will preserve the Fourier phase up to that diffraction limit. We use the colloquial name one-and-a-half-pass for this technique, in the sense that only the second pass is complete (i.e., both diffraction and aberrations do affect the PSF), whereas aberrations do not contribute to the first (half) pass retinal PSF. This technique allows us to obtain a direct and objective measurement of the PTF - up to the cutoff frequency of the first pass, diffraction-limited Gaussian-in the human eye (PTF estimates from aberroscopic data have been reported by Charman and $\mathrm{Walsh}^{10}$ ). In addition, the resulting aerial image constitutes a blurred (with an isotropic Gaussian blur) version of the true retinal PSF. We have obtained good results of aerial images, as well as PTF's, with both artificial and real eyes. When using appropriate entrance and exit pupils, the aerial image keeps most of the main features, overall shape, and structure of the retinal PSF. We propose two different ways to obtain an estimate of the true retinal PSF, including preliminary experiments: (a) deblurring the one-anda-half-pass aerial image (including results with off-axis data recorded on real eyes) and (b) combining the MTF obtained with conventional double pass with the oneand-a-half-pass PTF to retrieve the retinal PSF through Fienup's ${ }^{11}$ error reduction algorithm. The preliminary results are quite promising.

\section{METHODS}

The aerial image, $I\left(x^{\prime}, y^{\prime}\right)$, formed after double pass ${ }^{8}$ through the optical media of the eye, is the cross correla- 
tion of the two PSF's:

$$
I\left(x^{\prime}, y^{\prime}\right)=P_{1}(-x,-y) \otimes P_{2}\left(x^{\prime}, y^{\prime}\right)
$$

where $x, y$ and $x^{\prime}, y^{\prime}$ are spatial coordinates in the retinal plane and the aerial image plane, respectively, and $\otimes$ signifies convolution. In other words, the aerial image, $I\left(x^{\prime}, y^{\prime}\right)$, is the convolution of the inverted geometrical image of the first-pass retinal PSF with the secondpass PSF. In the conventional double pass, $P_{1}(x, y)=$ $P_{2}\left(x^{\prime}, y^{\prime}\right)$ by the reversion principle. ${ }^{12}$ In that case, the aerial image is the autocorrelation of the retinal singlepass image. Thus it is not possible to recover the retinal PSF (deconvolution would yield a sort of center symmetric version of the true PSF).

We can break such symmetry so that the cross correlation keeps some phase information, for example, by using different pupils for the two passages. In particular, we can choose a small pupil for the input beam, causing the retinal image to be diffraction limited and, more important, center symmetric. When an optical system is aberration free or only has even-symmetric aberrations (defocus, spherical aberration, astigmatism, etc.), the PSF is center symmetric. Consequently its corresponding PTF is flat (zero). By contrast, we can use a large exit pupil in the second pass: the larger the pupil for the outcoming beam, the larger the effect of odd-symmetric aberrations and the larger the asymmetry between the two passages. This is illustrated in Fig. 1. The diagram represents a lens with an off-axis object, so that the image displays coma. If we place a very small pupil in front of the lens (upper diagram), the effect of coma will be negligible compared with the effect of diffraction. The result will be a diffraction-limited Airy disk. Now, if we use a large exit pupil for the second pass, we get the convolution [Eq. (1)] of the inverted image of that Airy disk (which has radial symmetry) with the secondpass PSF. This second-pass PSF shows coma, just as the first-pass PSF would have shown if a larger entrance pupil had been used. The Fourier phase of the Airy disk is zero. Thus the phase of the Fourier transform of the aerial image directly provides the single-pass retinal PTF, up to the cutoff frequency of the Airy disk. As mentioned above, this is true not only for aberration-free Airy disks but also for PSF's affected only by even-symmetric aberrations.

The aerial image obtained with this one-and-a-half-pass method shows the effect of both even- and odd-symmetric aberrations. However, this is not yet the true retinal PSF, because the extra blurring, which is due to the convolution with the inverted Airy disk, also affects the resulting aerial image. To obtain the true singlepass retinal PSF, we would need to remove such blurring by some decorrelation procedure (the same as deconvolution with center-symmetric PSF's). In real in vivo eyes we cannot gain access to measure the retinal first-pass image, so we have to assume a PSF for this first pass. In this sense, it is especially useful to work with very small diffraction-limited pupils, which allows us to infer accurately the shape of the PSF, $\mathrm{P}_{1}$, needed for the deconvolution. The pupil size that guarantees diffraction-limited imaging can be obtained by the conventional double-pass method. A second interesting alternative to retrieve the retinal PSF is to combine the MTF, obtained by conventional double pass, with the PTF measured by one-and-a-half pass. Both deblurring and retrieval procedures are tested and discussed below.

Instead of using a circular stop as the entrance pupil, we have directly used an unexpanded Gaussian laser beam. This has two advantages: The experimental system is simpler, and we can use a much higher fraction of the light of the input beam. Also, this narrow Gaussian beam produces a diffraction-limited Gaussian (well behaved and easy to calibrate) that can significantly facilitate the deconvolution. This Gaussian PSF is the squared modulus of the Fourier transform of the Gaussian beam at its waist. $^{13}$

\section{A. Apparatus}

The experimental setup is depicted in Fig. 2 and is similar to the system used in conventional double-pass measurements. $^{2}$ The main difference is that now the illuminating beam, from a $\mathrm{He}-\mathrm{Ne}$ laser, is not expanded. Nevertheless, we clean up the beam with the help of a spatial filter. We have used two equal, symmetrically placed, confocal microscope objectives, with a pinhole $(25 \mu \mathrm{m})$ at the common focal point. Thus we filter the beam without expanding it. We calibrated the resulting Gaussian beam by measuring the location and size
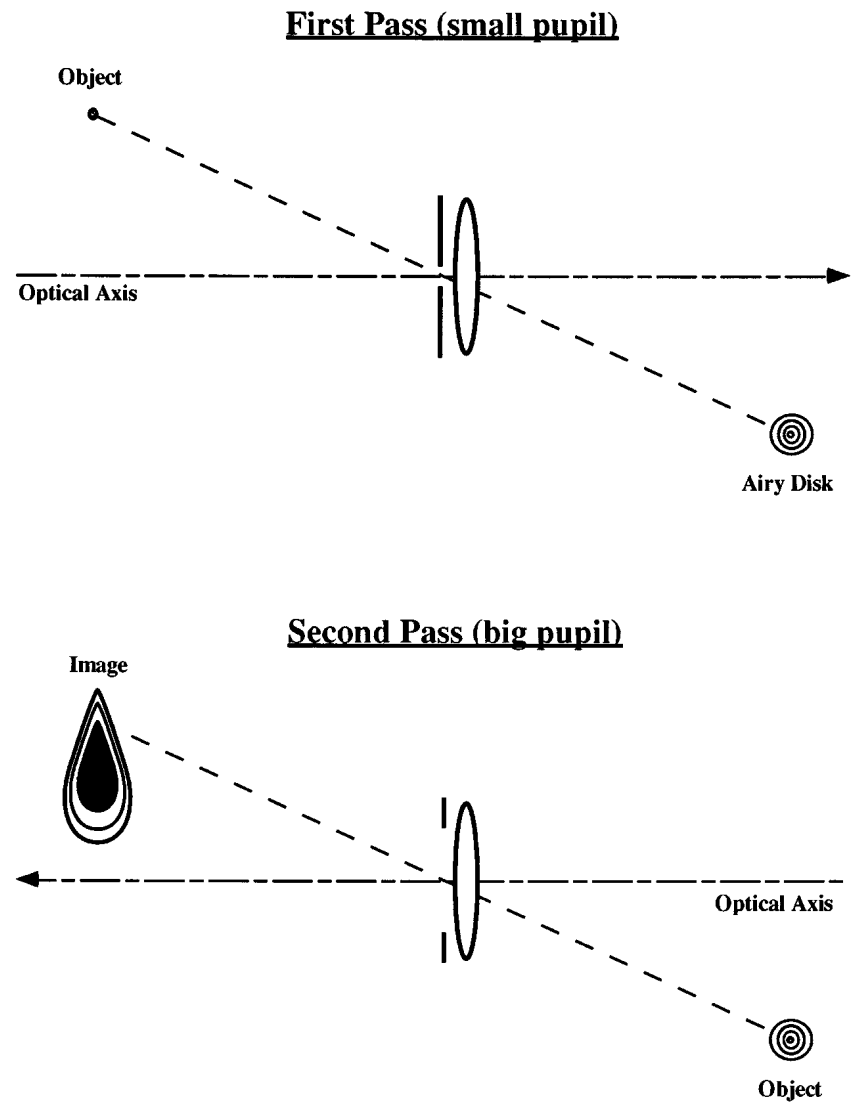

Fig. 1. Principle of the method. In the first pass (upper) the light from a point source (traveling from left to right) passes through a small pupil so that the image is a diffraction-limited Airy disk. Then, with a large pupil (lower) in the second pass (the light travels from right to left), the resulting image is the cross correlation between an aberrated second-pass image and the inverted image of the Airy disk. 


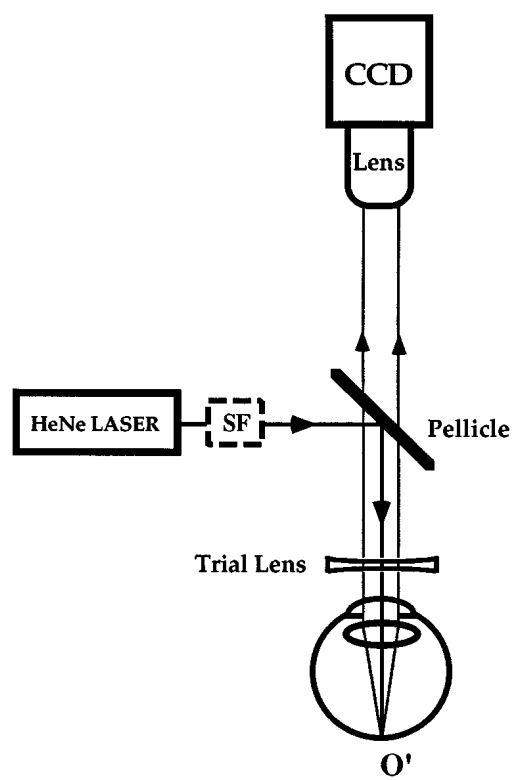

Fig. 2. Experimental setup. An unexpanded Gaussian beam $\left(w_{0}=0.4 \mathrm{~mm}\right)$ filtered with a spatial filter SF enters the eye after reflection off a pellicle and forms diffraction-limited image $\mathrm{O}^{\prime}$. Optional trial lenses compensate for sphere and cylinder refraction. After reflection off the retina, the beam passes through the full pupil and a 500-mm lens forms an aerial image on a CCD array.

of its waist, ${ }^{13} w_{0}=0.4 \mathrm{~mm}$. From these data we can estimate the diameter of the effective pupil, taken to be the diameter of the beam: $2 \sigma=0.58 \mathrm{~mm}$. The squared modulus of the Fourier transform of the beam profile at the waist gives us the radius of the Gaussian PSF at the focal plane (the retina). After reflection off a pellicle beamsplitter, the beam enters the eye through the center of the natural pupil, forming a diffraction-limited Gaussian PSF on the retina. The emerging beam (reflected off the retina) passes through the natural pupil (and through the pellicle) and is focused on a highresolution, cooled CCD array by a photographic objective $\left(f^{\prime}=500 \mathrm{~mm}\right)$. Optional trial lenses compensate for sphere and cylinder refractive errors of the eye.

For the artificial eye, we have modified the system to obtain up to four different configurations.

\section{B. Artificial Eye}

For preliminary testing of the method, we have conducted experiments with an artificial eye, composed of an $f^{\prime}=35 \mathrm{~mm}$ lens with an adjustable pupil and a rotating white diffuser placed at the focal plane. The artificial eye permits direct measurement of the single-pass PSF, which is important for testing our initial hypothesis and for calibrating the system. We recorded the PSF of the artificial eye in four different conditions. Two cases consisted of single-pass recordings with either small (Gaussian beam) or large pupil sizes. The two other cases were a conventional symmetric double pass with a large pupil and a one-and-a-half pass (with the setup described above). For the single-pass recordings we removed the white diffuser from the artificial eye and replaced it with the CCD camera now with a $20 \times$ microscope objective [similar to that depicted in Fig. 2(a) of Ref. 8]. With this single-pass configuration we obtained two PSF's, one with the small, 0.58-mm effective pupil (provided by the unexpanded beam) and another with a large, 4-mm pupil. For this large-pupil case, as well as for the conventional symmetric double pass, we used a conventional beam expander ${ }^{2}$ to fill the pupil completely. For the other two cases we used the double-pass configuration of Fig. 1 for small and large entrance pupils and placed the rotating diffuser at the focal plane of the artificial eye. In all four cases we carefully checked for focusing and scanned the refractive state by recording images with slightly different spherical refraction. We scanned the focus in $0.05-\mathrm{mm}$ steps (this corresponds to roughly half of the theoretical depth of focus,${ }^{14} 0.09 \mathrm{~mm}$, of the artificial eye) and selected the best image later.

We performed this procedure once with the lens of the artificial eye working on axis and once under off-axis conditions. In the latter case, instead of tilting the artificial eye, we de-centered the lens $3 \mathrm{~mm}$ with respect to the artificial pupil, since we previously experienced ${ }^{8}$ that this is a convenient method to obtain significant amounts of coma.

Our goal with this experiment was to test all our assumptions and to find the limits of this method, so we designed a complete and systematic experiment consisting of the following steps:

1. Record the single-pass PSF with small and large pupils. Then check for the diffraction-limited assumption: Compare the single-pass, small-pupil PSF with that predicted by the beam calibration.

2. Using direct computer simulation, predict doublepass PSF's (both symmetric and asymmetric) by autocorrelation or cross correlation, respectively, of single-pass data.

3. Record the double-pass (symmetric and asymmetric) PSF's and compare with predictions.

4. Compute the Fourier transform of the four recorded PSF's. Compare the PTF's obtained from the asymmetric double-pass data with single-pass data.

5. Decorrelate the double-pass and one-and-a-halfpass MTF's. Compare these estimates with single-pass data.

6. Reconstruct the single-pass PSF from the doublepass MTF and the one-and-a-half-pass PTF. Compare with single-pass data.

\section{RESULTS}

\section{A. Artificial Eye}

The six panels of Fig. 3 summarize the results corresponding to steps 1-3 above for the off-axis case, which is by far the most interesting. The upper row displays the images obtained with the unexpanded Gaussian beam (small pupil), whereas the lower row corresponds to those obtained with the expanded beam with a 4-mm pupil. The three columns show the single-pass images recorded with the microscope objective (step 1 , left); the aerial images recorded after two passages through the lens (step 3, middle); and the computer-simulated images (step 2, right) obtained by cross correlation of the two images of the left column (upper) and by autocorrelation of the image of the left (lower). 
The image in the upper-left panel is a diffraction limited, highly symmetric Gaussian. We have verified the diffraction-limited hypothesis by comparing the size of this image, $11.9 \mu \mathrm{m}$ (obtained with the unexpanded beam), with the theoretical prediction from the waist calibration, $12 \mu \mathrm{m}$. The agreement is excellent and within our experimental measuring error. The lower-left image presents a considerable amount of coma, which appears to be almost the only aberration present. We have estimated the tangential, $C_{T}=16^{\prime}$ (minutes of arc), and sagital, $C_{S}=7.4^{\prime}$ coma.

The image in the upper-center panel shows a comalike shape, but it is not as pure as the lower-left image. Extra blurring is clearly present in comparison with the singlepass PSF (lower left). This effect agrees well with the predicted result (upper right). The image below (lower center) has a characteristic bow-tie shape, also as predicted (lower right). The similarity between the central column with experimentally recorded data and the simulated data (right) is remarkable, which supports our departure hypothesis.

We have tried to measure the amount of geometrical coma from the one-and-a-half-pass aerial image, but the extra blurring due to the correlation with the Gaussian will cause some biased estimates. We have obtained the values $C_{T}=18.2^{\prime} ; C_{S}=6.9^{\prime}$; i.e., we have overestimated the tangential coma and slightly underestimated the sagittal coma. The bias is not large, although it will depend on the balance between the amount of real aberration and the additional diffractional blurring.

Figure 4 compares the single-pass PTF (left) with that obtained after one and a half pass. In both cases we have applied a phase-unwrapping algorithm ${ }^{15}$ and substracted the linear term. The linear phase is related to lateral shifts of the whole PSF and thus has no influence on its shape. ${ }^{10}$ There is a good agreement up to the effective cutoff frequency of the diffraction-limited Gaussian. (Minor discrepancies are due mainly to the different accuracy in estimating and substracting the linear phases in both cases.) We have considered that the effective cutoff is the frequency at which the noise dominates the PTF so that it loses smoothness - here $\sim 75$ cycles per degree (c/deg) (vertical) and $\sim 50 \mathrm{c} / \mathrm{deg}$ (horizontal) for the single-pass case and roughly half of these values for the one-and-a-half-pass case. Figure 5 compares the MTF's obtained in the four recordings. The curves correspond to radial profiles of the two-dimensional functions. The single-pass MTF (continuous curve) is our reference. The dashed curve is the double-pass MTF (the square root of the Fourier transform of the aerial image). The agreement between the single- and double-pass MTF's is almost perfect, which validates the double-pass approach. The curve with the open squares is an early attempt to decorrelate the one-and-a-half-pass aerial image. A reasonable agreement has been obtained up to $20 \mathrm{c} / \mathrm{deg}$, which could be further improved with a more exact deconvolu-
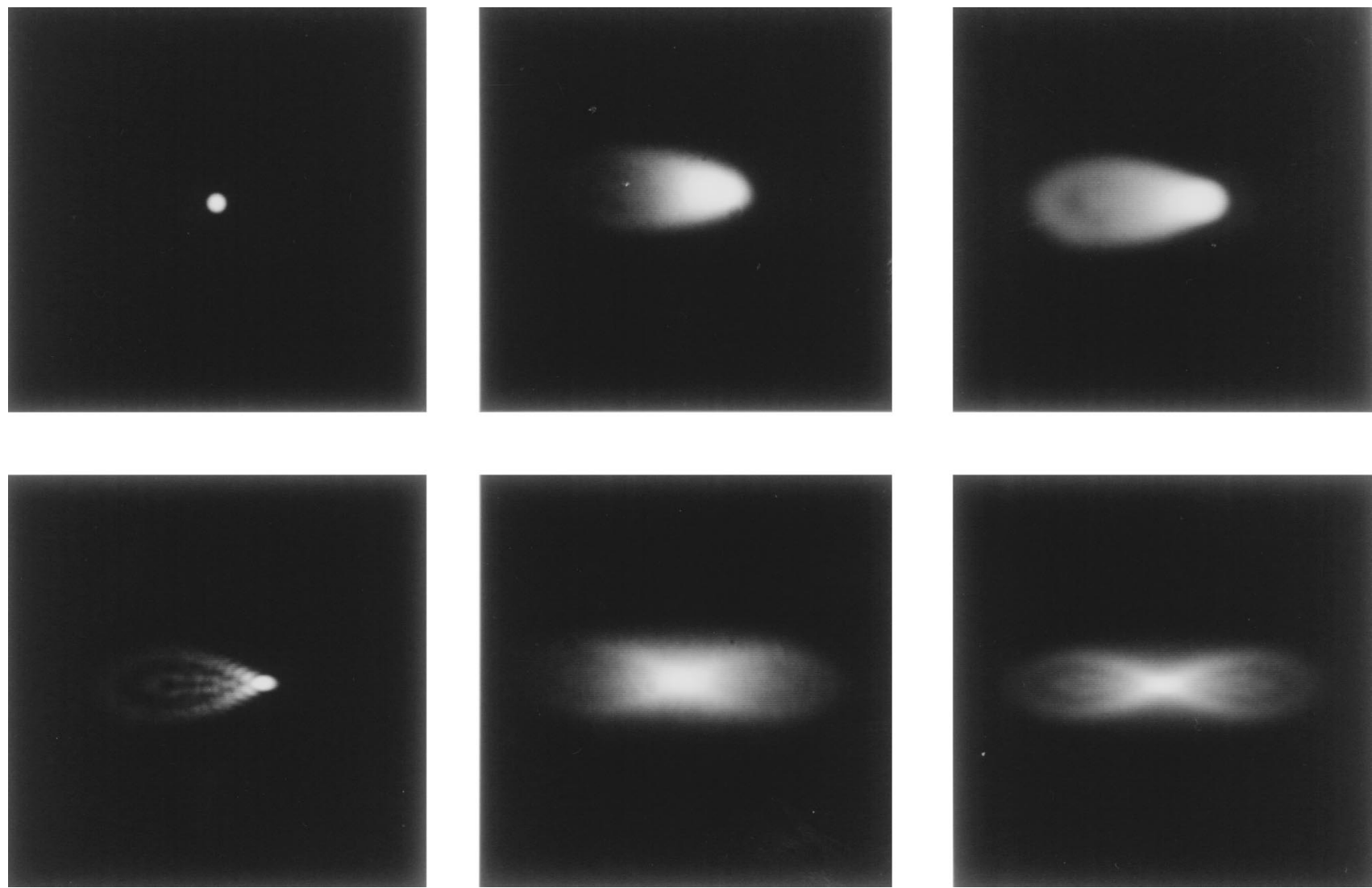

Fig. 3. Results obtained with an artificial eye. The images in the upper row were obtained with an unexpanded Gaussian beam; those in the lower row were obtained with an expanded beam and a 4-mm pupil. The two panels in the left column show the resulting single-pass images. The two panels in the center column were recorded after reflection off a diffuser and a second passage through the lens. The two panels in the right column are the computed cross correlation between the images of two left panels (upper row) and the autocorrelation of the image of the lower-left panel (lower row), respectively. 

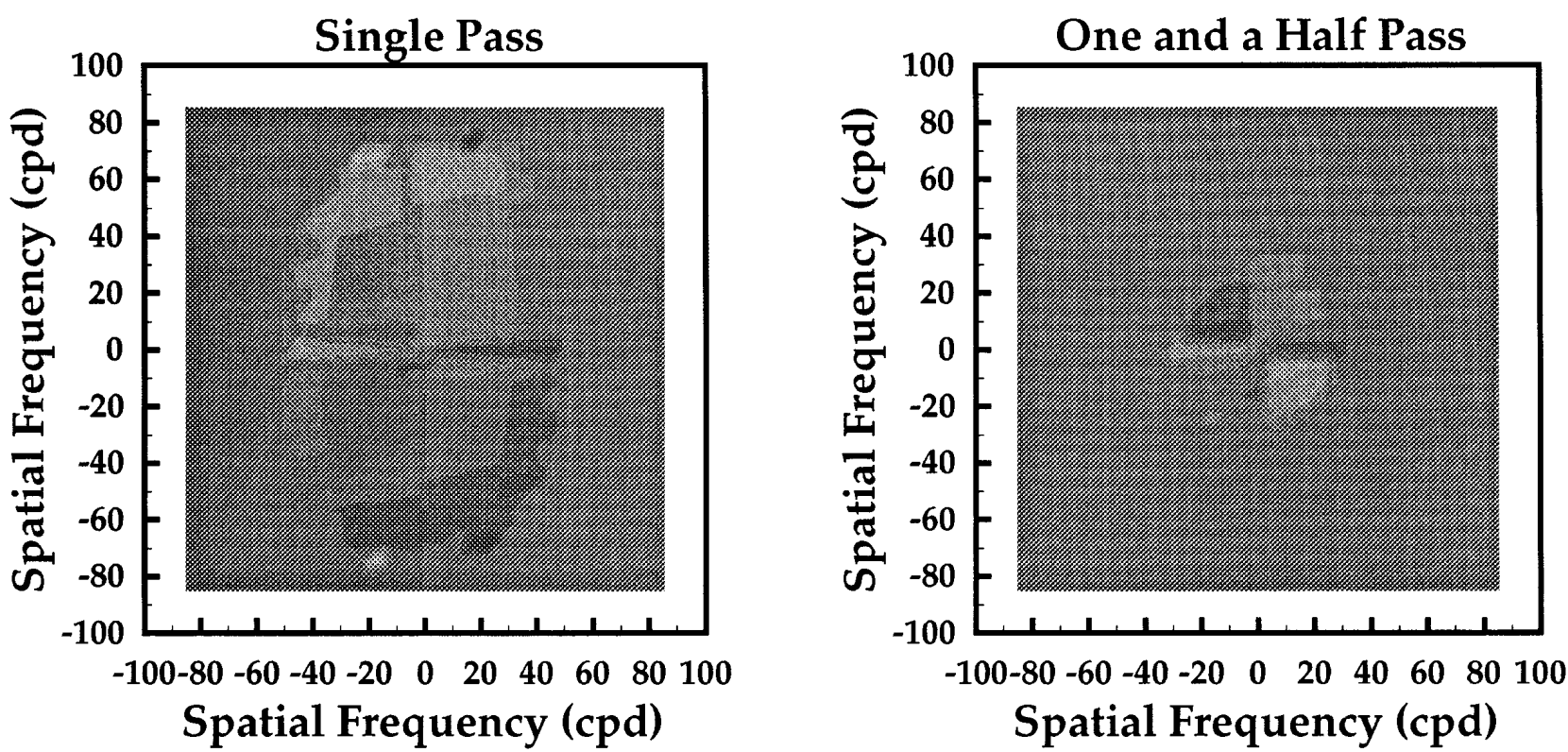

Fig. 4. Comparison of the single-pass PTF (left) with the one-and-a-half-pass estimate (right). Changes in gray level correspond to phase increments of $\pi$; middle gray signifies zero phase, and darker and lighter levels denote negative and positive phase, respectively. The agreement is good up to the diffraction limit of the first-pass Gaussian PSF, except for remaining linear offsets. In this and subsequent figures, cpd stands for c/deg.

tion method. However, this one-and-a-half-pass method will hardly provide the MTF for frequencies higher than the cutoff of the single-pass Gaussian (closed circles). A possible solution is to use the conventional double pass to obtain the MTF and the one-and-a-half pass for the PTF as shown in Fig. 6. We obtained the reconstruction of the PSF, in the right-hand panel, with Fienup's iterative Fourier transform algorithm. ${ }^{11}$ This is a preliminary attempt in which we imposed the known doublepass MTF as the Fourier domain constraint, whereas the one-and-a-half-pass aerial image was the input guess. In this way, the algorithm helps to deblur the aerial image, extrapolating the unknown high-frequency phase while practically not changing the known phase. The results obtained with this crude approach (right-hand panel) are in close agreement with the single-pass PSF (left-hand panel).

So far we have investigated with an artificial eye the possibilities of this new technique, alone or combined with the conventional double-pass method. Next, we present results obtained with the real eye.

\section{B. Human Eye}

Figure 7 shows results obtained with this new one-and-ahalf-pass technique. They correspond to three observers, two different visual angles - the fovea or visual axis (upper row) and $25^{\circ}$ temporal eccentricity (lower row). The observers viewed a fixation target (the point source in the foveal case and a bright spot in the off-axis case ${ }^{2}$ ) with natural pupil and accommodation. Head position was fixed by a bite bar with an $X Y$ micrometric translation stage. Careful alignment of the center of observer's pupil with the input beam was established in both axes. We compensated for the refractive state of the observers (both sphere and cylinder) with trial lenses for the foveal recordings to obtain the best image quality, whereas the off-axis images were obtained with the naked eye, and therefore astigmatism or some defocus may be present. The pupil size was monitored by an infrared TV camera. ${ }^{2,4}$ The sphere $S$ and cylinder $C$ refraction (in diopters) and pupil diameter $\phi$ (in millimeters) for the three observers were ON ( $S=0, C=0, \phi=5)$; MA ( $S=-1.25, C=-0.5$ axis $\left.85^{\circ}, \phi=4.5\right)$; $\mathrm{RN}\left(S=-0.5, C=-0.75\right.$ axis $\left.0^{\circ}, \phi=3.8\right)$. The upper row of Fig. 7 shows three images nearly astigmatism free (as we would expect after cylinder compensation). These images present different degrees of symmetry, from the nearly complete rotational symmetry for RN to some odd-symmetric and irregular features for observer ON. MA's aerial image would correspond to an intermediate case. The smoothness of these images is probably due to the additional diffraction blurring, which can potentially mask important features or structures. For the off-axis images (bottom row), however, observer ON shows a roughly even-symmetric pattern dominated by astigmatism, while $\mathrm{RN}$ has a characteristic comashaped image. For this observer, the claim that astigmatism is the most important off-axis aberration ${ }^{2}$ does not

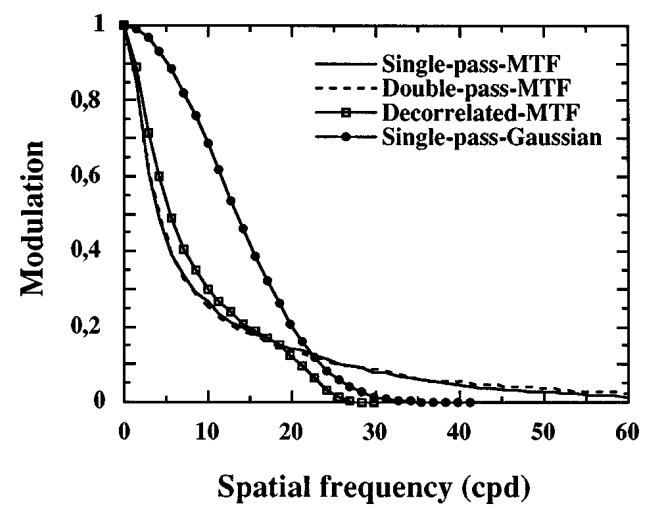

Fig. 5. Radial profiles of the MTF's obtained from the aerial images of Fig. 3 (artificial eye). The match between the singlepass and double-pass MTF's is very good. With this novel technique, decorrelation is possible up to the effective cutoff frequency of the first-pass, diffraction-limited image. 

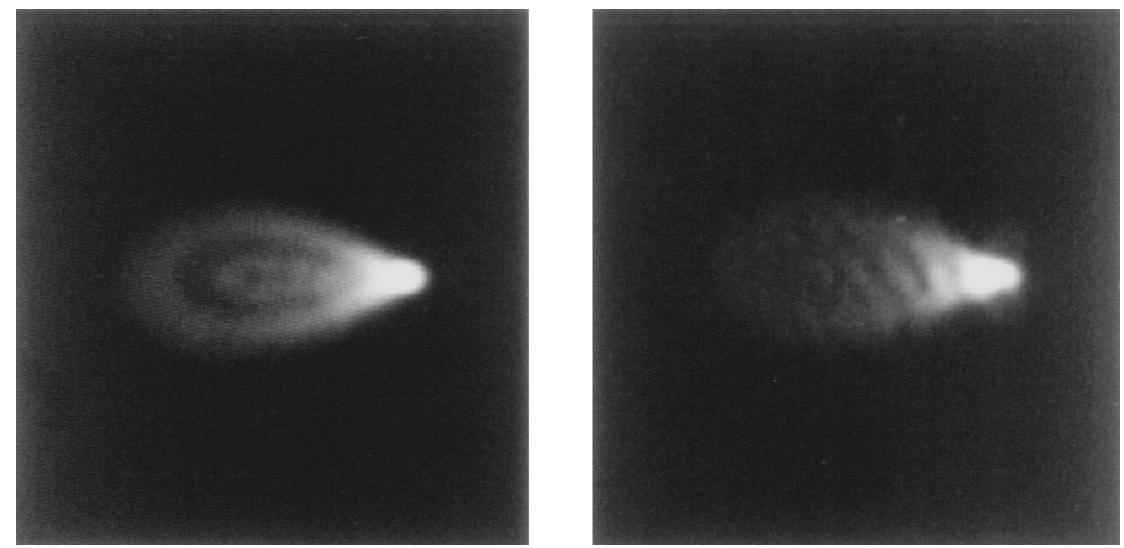

Fig. 6. Reconstruction of the first-pass PSF (right) from the double-pass MTF and one-and-a-half-pass PTF data. The left panel shows the directly recorded single-pass PSF for comparison.
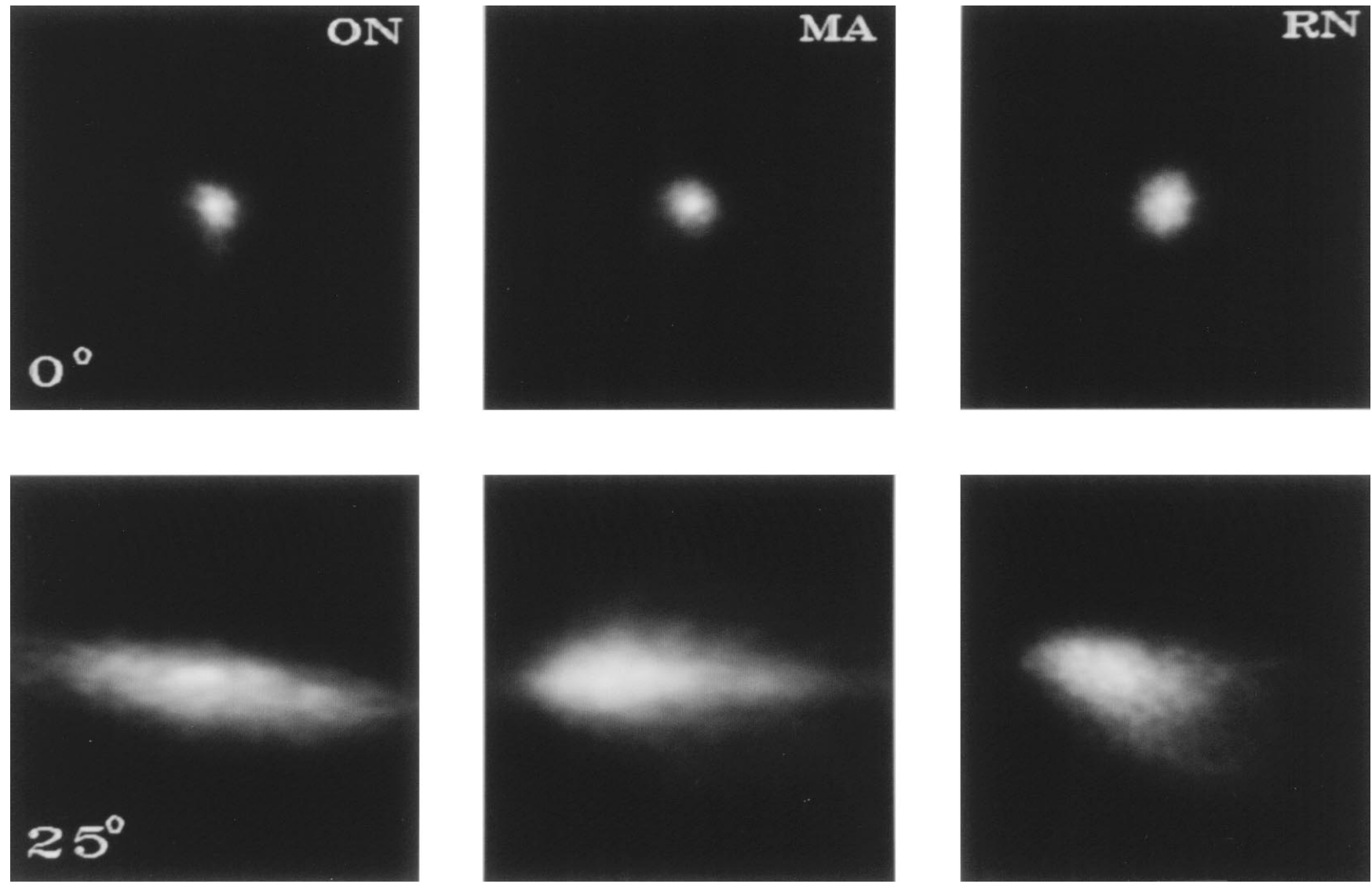

Fig. 7. Aerial images for three observers (ON, MA, and RN), for $0^{\circ}$, fovea (upper row), and $25^{\circ}$, peripheral retina (lower row). They show different amounts of astigmatism (even symmetric), coma (odd symmetric), and irregular aberration.

hold. Figure 8 compares the PTF's for $0^{\circ}$ and $25^{\circ}$ eccentricities for observer MA. The lack of shallowness suggests the presence of odd-symmetric abberations in both cases, although the off-axis PTF suggests much larger aberrations. Consequently the off-axis PTF is steeper and the effective cutoff frequency is much smaller for the vertical direction $(\sim 20 \mathrm{c} / \mathrm{deg})$. The effective cutoff frequency is $\sim 40 \mathrm{c} / \mathrm{deg}$ for the fovea. In the off-axis case aberrations clearly dominate over first-pass diffraction, which suggests that decorrelation may be possible here. We have roughly estimated the amount of coma for this observer, resulting in $C_{T}=32.3^{\prime} ; C_{S}=4.3^{\prime}$. (Note, how- ever, that the data analysis made with the artificial eye suggests that we would be overestimating somewhat the actual $C_{T}$ while underestimating $C_{S}$.) The image obtained from subject MA again represents an intermediate case containing even (astigmatism), odd-symmetric (coma), and irregular aberrations.

\section{DISCUSSION}

The results obtained with both artificial and real eyes seem to confirm that, for large amounts of odd-symmetric and irregular aberration, the asymmetric double-pass 
method permits us to obtain aerial images with a more realistic symmetry and structure than those provided by the symmetric double-pass method. In this sense, the off-axis images shown here are closer to the true shape of the retinal PSF than those aerial images previously published. $^{2}$ These images show the effect of all types of aberrations: even, odd, and irregular aberrations. However, Eq. (1) clearly states that the aerial image is the correlation between two functions. We have chosen one of these two functions to be a rotationally symmetric, diffraction-limited Gaussian, which actually allows us to retain the Fourier phase information in the second-pass image. However, we have found potential problems or drawbacks associated with the breaking of the symmetry in the double pass. One preliminary question is whether the first pass, with the Gaussian beam, will always form a diffraction-limited image. We have recorded single-pass images of this Gaussian beam with $0.58-\mathrm{mm}$ effective diameter and different amounts of decentration (we used $3 \mathrm{~mm}$ of decentration to obtain the images of Fig. 3). For large decentrations ( $4 \mathrm{~mm}$ or more), the PSF started to show some elongation. The image was a sort of elliptical
Gaussian because of astigmatism. Consequently, one has to take care when working at large eccentricities, as the more eccentric the field, the narrower the input beam must be (though we can always compensate for astigmatism with the help of trial lenses). If we are interested mainly in the PTF (for instance, when obtaining the MTF with a conventional double-pass system), the aberrationfree condition can be greatly relaxed. In this case the first-pass image has to be free of odd-symmetric and irregular aberrations. Defocus, astigmatism, or spherical aberration can be tolerated, since they do not contribute to the PTF.

Possibly the most important drawback of the one-anda-half-pass method is the low cutoff frequency imposed by the use of such a small effective pupil. For our $\mathrm{He}-\mathrm{Ne}$ laser $(638 \mathrm{~nm})$, the effective cutoff frequency for that Gaussian beam is (see Figs. 4 and 5) of the order of $30 \mathrm{c} / \mathrm{deg}$, which is far from the optical resolution of the eye. ${ }^{2,5}$ In the upper row of Fig. 7, the first-pass Gaussian seems to dominate the overall shape of the images, which tends to mask irregular features of the true retinal image. Therefore, for the results presented here,
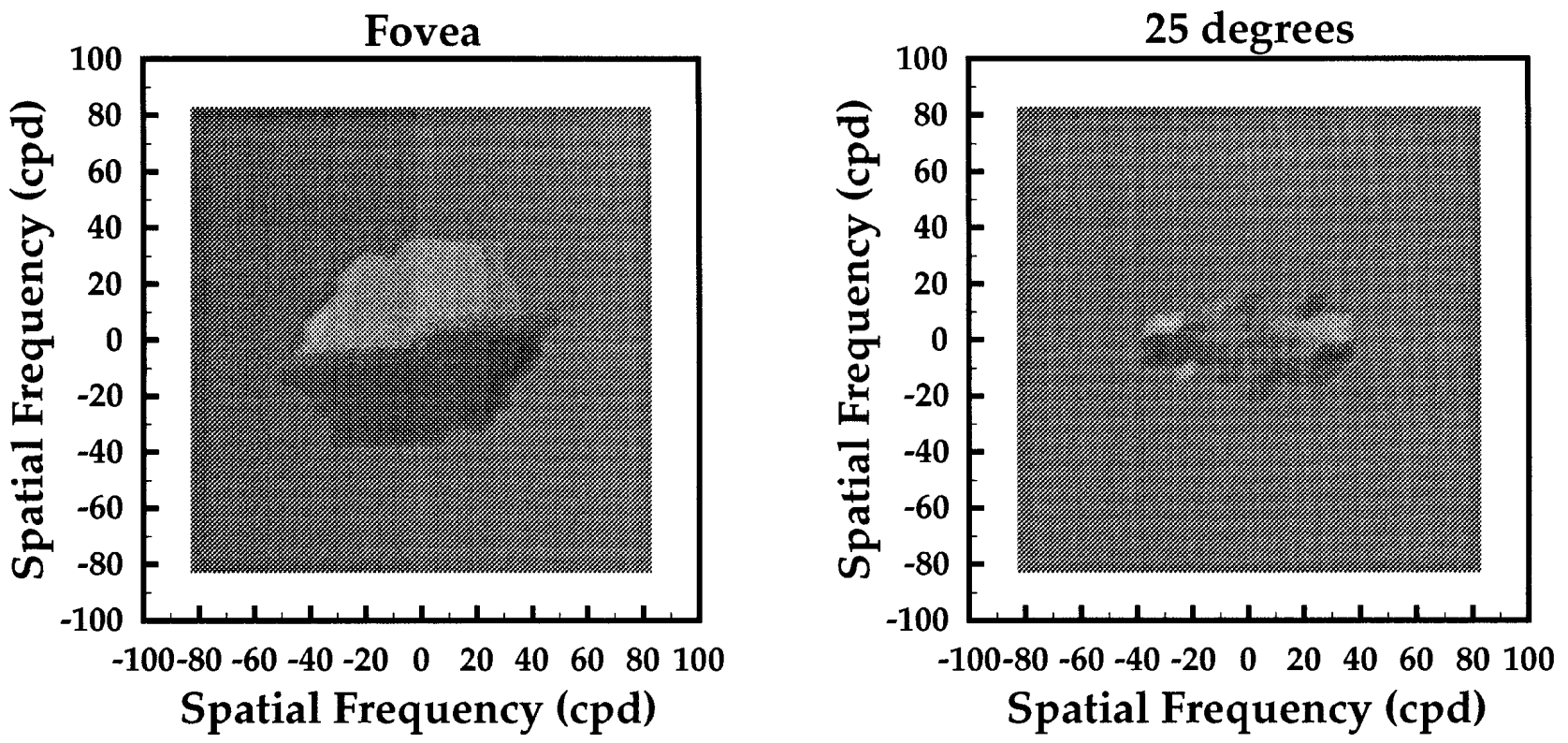

Fig. 8. Phase transfer functions for observer MA, for $0^{\circ}$ (left), and $25^{\circ}$ (right) eccentricity. Changes in gray level correspond to phase increments of $\pi$; middle gray stands for zero phase, and darker and lighter levels mean negative and positive phases, respectively. They differ both in the effective cutoff frequency and the steepness owing to the larger aberrations in the off-axis case.
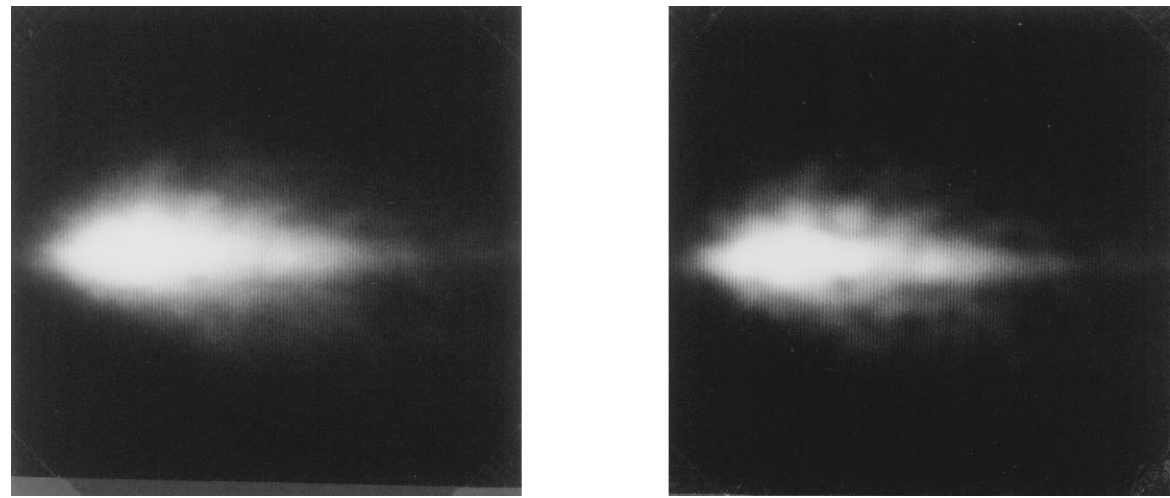

Fig. 9. Decorrelated version (right) of the one-and-a-half-pass aerial image (left) of observer MA, for $25^{\circ}$ of eccentricity. Image features, including noise, are sharper after decorrelation. 
especially for the foveal data, a complete decorrelation is not possible. For the off-axis case, however, the large aberrations make the shape of the true retinal image dominate the first-pass Gaussian, so that the aerial image reveals most of the real features of the retinal PSF (though they are somewhat smoothed by correlation with the Gaussian). Thus, for the off-axis case, it is worthwhile to attempt such decorrelation; Fig. 9 shows our result for observer MA. The decorrelation provides sharper features but also enhances the noise. In this particular case (as well as for the other PSF's at this eccentricity) the result is a noisy, but close to the true shape, estimate of the retinal PSF. Since the exact decorrelation may not be possible in general owing to the limited bandwidth imposed by diffraction, a partial solution for this problem would be to use a larger beam diameter when needed. For the fovea (and parafovea), where aberrations are not as large as peripherally, we could double the diameter of the input beam while keeping the retinal image nearly diffraction limited. ${ }^{3}$ This would permit decorrelating the aerial image up to roughly $50 \mathrm{c} / \mathrm{deg}$. Another way to obtain better results is to use larger exit pupils, which increases the asymmetry between the two passes. (We have tested pupils between 4 and $5 \mathrm{~mm}$ ). With larger pupils the aberrations increase (to values close to those found in the off-axis case) and the decorrelation is easier.

A more attractive possibility is to combine this technique with the standard double-pass method. Figure 5 clearly shows that the double pass (i.e., taking the square root of the Fourier transform of the aerial image) provides a very good estimate of the single-pass MTF. The present asymmetric method directly provides the PTF up to the cutoff frequency of the first-pass Gaussian PSF. To obtain the true retinal PSF (see Fig. 6), we would need to extrapolate the known phase (for the lower frequencies) to estimate the unknown phase (higher frequencies). We will continue investigating and improving the reliability of such partial phase retrieval in further work. Preliminary results suggest that knowing the phase for the lower frequency range will make the task much easier than in a typical phase-retrieval problem, ${ }^{11}$ in which no phase information (and no good input guess) is available. In addition, we think that the low-frequency phase information would be more important than high-frequency phase information in this kind of image, because the phase of the lower frequencies governs the global shape of the image (coarse details, main symmetries, etc.), i.e., loworder aberrations. In contrast, the phase of the high frequencies governs fine details (i.e., high-order aberrations) and is much more affected by noise. However, it does have a minor influence on the overall shape of the PSF. This problem is more general, because measuring high-order aberrations is always difficult, even with wavefront sensors. ${ }^{7}$ Finally, our preliminary data-although restricted to a small sample of observers-indicate the presence of all types of aberration (astigmatism, coma, and irregular, as previously reported by Howland and Howland ${ }^{9}$ ) mainly off axis, suggesting that there is no common aberration pattern among individuals. Astigmatism often seems to be the dominant aberration, but we have found at least one PSF ( $\mathrm{RN}, 25^{\circ}$ eccentric) with a shape that is clearly dominated by coma.

\section{ACKNOWLEDGMENTS}

This research was supported by the Comisión Interministerial de Ciencia y Tecnología (Spain) under grant TIC940849. We thank Oscar Nestares for his patience and help.

\section{REFERENCES}

1. M. F. Flamant, "Etude de la repartition de la lumière dans l'image rétinienne d'une fente," Rev. Opt. 34, 433-459 (1955).

2. R. Navarro, P. Artal, and D. R. Williams, "Modulation transfer of the human eye as a function of retinal eccentricity," J. Opt. Soc. Am. A 10, 201-212 (1993).

3. P. Artal and R. Navarro, "Monochromatic modulation transfer function of the human eye for different pupil diameters: an analytical expression," J. Opt. Soc. Am. A 11, 246-249 (1994).

4. R. Navarro, M. Ferro, P. Artal, and I. Miranda, "Modulation transfer functions of eyes implanted with intraocular lenses," Appl. Opt. 32, 6359-6367 (1993).

5. D. R. Williams, D. Brainard, M. McMahon, and R. Navarro, "Double pass and interferometric measures of the optical quality of the eye," J. Opt. Soc. Am. A 11, 3123-3135 (1994).

6. J. Liang and D. R. Williams, "Effect of higher order aberrations on image quality in the human eye," in Vision Science and Its Applications, Vol. 1 of 1995 OSA Technical Digest Series (Optical Society of America, Washington, D.C., 1995) pp. $70-73$.

7. J. Liang, B. Grimm, S. Goelz, and J. F. Bille, "Objective measurement of wave aberrations of the human eye with the use of a Hartmann-Shack wave-front sensor," J. Opt. Soc. Am. A 11, 1949-1957 (1994).

8. P. Artal, S. Marcos, R. Navarro, and D. Williams, "Odd aberrations and double pass measurements of retinal image quality," J. Opt. Soc. Am. A 12, 195-201 (1995).

9. H. C. Howland and B. Howland, "A subjective method for the measurement of the monochromatic abberations of the eye," J. Opt. Soc. Am. 67, 1508-1518 (1977).

10. W. N. Charman and G. Walsh, "The optical phase transfer function of the eye and the perception of spatial phase," Vision Res. 25, 619-623 (1985).

11. J. R. Fienup, "Reconstruction of an object from the modulus of its Fourier transform," Opt. Lett. 3, 27-29 (1978).

12. M. Born and E. Wolf, Principles of Optics, 6th ed. (Pergamon, Oxford, 1980)

13. B. E. A. Saleh and M. C. Teich, Fundamentals of Photonics (Wiley, New York, 1991).

14. W. J. Smith, Modern Optical Engineering (McGraw-Hill, New York, 1966)

15. D. C. Ghiglia, G. A. Masting, and L. A. Romero, "Cellularautomata method for phase unwrapping," J. Opt. Soc. Am. A 4, 267-280 (1987). 